\title{
UM PROCEDIMENTO PARA O CÁLCULO DO ÍNDICE DE DIVERSIDADE DE BRILLOUIN ${ }^{1}$
}

\author{
Renato R.C. Dutra ${ }^{2}$
}

\begin{abstract}
A PROCEDURE TO CALCULATION OF BRILlOUIN'S DIVERSITY INDEX. The purpose of this note is to make possible the obtainment of common logarithm of factorial values above 499, making feasible the utilization of Brillouin's Diversity Index.

KEY WORDS. Ecological Index, Alpha Diversity. Brillouin Index
\end{abstract}

Sendo um dos mais utilizados (cf. FAZEKAS et al. 1992; DAVIS 1993; WAHLBRINK \& ZUCCHI 1994; KAPPELLE et al. 1995, entre outros) para se calcular a diversidade alfa (sensu WHITTAKER 1972), o Índice de Diversidade de Shannon tem as seguintes características: 1) O H' de Shannon só é definido para condições médias de uma população grande (Goldman apud PiElou 1966a) e; 2) O valor de $\mathrm{H}^{\prime}$ está baseado nas proporções das várias espécies de uma população de tamanho desconhecido, não podendo ser calculado com exatidão, mas apenas estimado (PIELOU 1966b).

Segundo MagurRan (1988: 34; $c f$. Pielou 1966b) este Índice assume que todas as espécies estão representadas na amostra, e que os indivíduos foram amostrados aleatoriamente de uma população de tamanho desconhecido.

Porém, quando a aleatoriedade da amostra não pode ser garantida, como durante amostragens de insetos com armadilhas luminosas, ou se a comunidade foi totalmente identificada, com todos os indivíduos contados, o Índice de Brillouin ( $c f$. NitTa 1991; Campos \& Carbonell 1994; Monteforte \& GarciagasCa 1994; ROUSE \& EVANS 1994; ANDRES \& WITMAN 1995, entre outros) é a forma apropriada a ser utilizada (MAgurRAn 1988: 37; PIELOU 1966a, 1966b), assim calculado: $\mathrm{HB}=\frac{\ln \mathrm{N} !-\sum \ln \mathrm{n}_{\mathrm{i}} !}{\mathrm{N}}$.

Entretanto, a maior dificuldade encontrada para a utilização do Índice de Brillouin está na obtenção do logaritmo natural do fatorial de valores acima de 69 , já que o cálculo deste valor é o limite da maioria das calculadoras e microcomputadores.

1) Contribuição número 924 do Departamento de Zoologia, Universidade Federal do Paraná.

2) Departamento de Zoologia. Universidade Federal do Paraná. Caixa Postal 19020, 81531-990 Curitiba, Paraná, Brasil. Bolsista do CNPq. 
Uma alternativa é a utilização de Tabelas Estatísticas, como a de Pearson \& Hartley (apud PIElou 1966b) que vai até 1000, ou a de RoHLF \& SOKAL (1981), e que apresenta o logaritmo de base 10 de fatoriais que vão de zero a 499; estes valores sendo muito baixos para aqueles que trabalham com insetos, por exemplo.

Assim, o objetivo deste trabalho é apresentar uma alternativa que permite o cálculo aproximado do logaritmo de base 10 para valores acima de 499, quando o acesso às Tabelas de Rohlf \& Sokal e de Pearson \& Hartley não for possível, ou quando for necessário trabalhar com valores acima de 1000.

Exemplo. Para os números 44 e 67, a Tabela de Rohlf \& Sokal apresenta os seguintes valores: $\log 44 !=54,4246$ e $\log 67 !=94,5619 ; 54,4246 / 94,5619=$ 0,576 .

\section{Aproximação de Stirling para $\mathrm{n}$ !}

Segundo SPIEgEL (1976: 169) quando "n" é grande e a avaliação direta de n! é impraticável, deve-se usar uma fórmula aproximada, devida a Stirling, a saber: $\mathrm{n} ! \sim \sqrt{2 \pi \mathrm{n}} \mathrm{n}^{\mathrm{n}} e^{-n}$ onde " $e$ " é a base dos logaritmos naturais.

\section{Exemplos:}

1) 44 ! $\sqrt{2 \Pi(44)} 44^{44} e^{-44}=\mathrm{S}$

Para o cálculo de $\mathrm{S}$, empregam-se os logaritmos de base 10. Então:

$\log \mathrm{S}=\log \sqrt{88 \pi} 44^{44} e^{-44}=$

$=1 / 2 \cdot \log 88+1 / 2 \cdot \log +44 \cdot \log 44-44 \cdot \log \mathrm{e}=$

$=1 / 2 \cdot \log 88+1 / 2 \cdot \log 3,142+44 \cdot \log 44-44 \cdot \log 2,718=$

$=1 / 2(1,9445)+1 / 2(0,4972)+44(1,6435)-44(0,4342)=$

$=0,9723+0,2486+72,3140+19,1048=$

$=54,4301$

Logo, $S=3,04 \times 10^{54,4301}$

2) $67 ! \sim \sqrt{2 \pi(67)} 67^{67} e^{-67}=\mathrm{S}$

$$
\begin{aligned}
& \log \mathrm{S}=\log \sqrt{134 \pi} 67^{67} e^{-67}= \\
& =1 / 2 \cdot \log 134+1 / 2 \cdot \log +67 \cdot \log 67-67 \cdot \log e= \\
& =1 / 2 \cdot \log 134+1 / 2 \cdot \log 3,142+67 \cdot \log 67-67 \cdot \log 2,718= \\
& =1 / 2(2,1271)+1 / 2(0,4972)+67(1,8261)-67(0,4342)= \\
& =1,0636+0,2486+122,3487+29,0914= \\
& =94,5695 \\
& \text { Logo, } \mathrm{S}=3,04 \times 10^{94,5695}
\end{aligned}
$$

Assim, como 3,04 e 10 são valores constantes na expressão, são os números obtidos pelo método de Stirling (no caso 54,4301 e 94,5695) que deverão substituir os valores de $\ln \mathrm{N}$ ! ou $\ln \mathrm{n}_{\mathrm{i}}$ ! na fórmula de Brillouin.

Note-se que são valores muito próximos àqueles obtidos por RoHLF \& 
SOKAL (1981); o resultado da divisão $54,4301 / 94,5695=0,576$, ou seja, as relações permanecem.

Não é o objetivo desta nota discutir o fato das populações serem de tamanho conhecido ou não, se todas as espécies estão representadas na amostra, assim como o problema que envolve a seletividade ou não por parte do aparato de amostragem. Apenas parece ser conveniente a utilização dos Índices de Shannon e Brillouin, concomitantemente, quando da avaliação da Diversidade alfa de uma ou mais localidades amostradas.

WHITTAKER (1972) prefere a utilização do Índice de Diversidade de Shannon, ao de Brillouin, devido às suas propriedades para o tratamento de dados ecológicos. Já LAXTON (1978) sugere que medidas de Diversidade são desenvolvidas a partir de princípios axiomáticos, independentes de quaisquer considerações estatísticas, observando que, neste aspecto, o Índice de Brillouin é o que melhor satisfaz a estes axiomas, se comparado ao de Shannon.

\section{REFERÊNCIAS BIBLIOGRÁFICAS}

ANDRES, N.G. \& J.D. WitMAN. 1995. Trends in community structure on a jamaican reef. Marine Ecology 118 (1-3): 305-310.

CAmpos, A. \& E. CARBOnell. 1994. Parasite community diversity in two Mediterranean labrid fishes Symphodus tinca and Labrus merula. Jour. Fish Biol. 44 (3): 409-413.

DAVIS, A.L.V. 1993. Alpha-diversity patterns of dung beetle assemblages (Coleoptera: Scarabaeidae, Aphodiidae, Staphylinidae, Histeridae, Hydrophilidae) in the winter rainfall region of South Africa. African Entomology 1 (1): 67-80.

FazeKas, J.; F. Kadar \& G.L. Lovei. 1992. Comparison of ground beetle assemblages (Coleoptera: Carabidae) of an abandoned apple orchard and the bordering forest. Acta Phytopathologica et Entomologica Hungarica 27 (1-4): 233-238.

Kappelle, M.; P.A.F. Kennis \& R.A.J. DE VRIES. 1995. Changes in diversity along a successional gradient in a costa rican upper montane Quercus forest. Biodiversity \& Conservation 4 (1): 10-34.

LaXTON, R.R. 1978. The measure of diversity. J. Theor. Biol. 70: 51-67.

MAgurRAn, A.E. 1988. Ecological Diversity and its measurement. New Jersey, Princeton University Press, 179p.

Monteforte, M. \& A. Garciagasca. 1994. Spat collection studies on pearl oysters Pinctada mazatlanica and Pteria sterna (Bivalvia, Pteriidae) in Bahia de La Paz, South Baja California, Mexico. Hydrobiologia 291: 21-34.

NITTA, T. 1991. Diversity of root fungal floras:its implications for soil-borne diseases and crop growth. Japan Agricultural Research Quaterly 25: 6-11.

PIELOU, E.C. 1966a. The measurement of diversity in different types of biological collections. J. Theor. Biol. 13: 131-144.

1966b. Shannon's formula as measure of specific diversity: it's use and 
misuse. Amer. Nat. 100 (914): 463-465.

RohlF, F.J. \& R.R. SoKal. 1981. Statistical Tables. New York, W.H. Freeman and Company, 219p.

Rouse, A.J. \& J.G. Evans. 1994. Modern land Mollusca from Maiden Castle, Dorset, and their relevance to the interpretation of subfossil archaeological assemblages. Jour. Mollusc. Stud. 60 (3): 315-329.

SpIEgel, M.R. 1976. Estatística. São Paulo, Ed. McGraw-Hill do Brasil, 580p.

WAHLBRINK, D. \& H. ZuCCHI. 1994. Occurrence and settlemmant of carabid beetles on an urban railway embankment - a contribution to urban ecology. Zoologische Jahrbucher, Systematik, Okologie und Geographie der Tiere 121 (2): 193-201.

WhitTAKer, R.H. 1972. Evolution and measurement of species diversity. Taxon 21: 213-251. 